\title{
Die Einführung der leistungsbezogenen Fallpauschalen zu verschieben, ist nicht nur gesetzeswidrig, sondern auch überflüssig
}

\section{Pierre-François Cuénoud}

Mitglied des FMH-Zentralvorstands, Verantwortlicher Ressort SwissDRG
Korrespondenz:

Dr. med. Pierre-François Cuénoud

FMH

Elfenstrasse 18

CH-3000 Bern 15

Tel. 0313591111

Fax 0313591112
Die «Akademie Affoltern» ruft in einer Petition dazu auf, die Einführung einer einheitlichen nationalen Tarifstruktur mit Pauschalen vom Typ DRG aufzuschieben. Dies wirkt befremdlich, da in $3 / 4$ der Kantone bereits ein solches System existiert und nur noch drei Kantone ihre Spitalleistungen anhand von Tagespauschalen verrechnen. In Wirklichkeit ist die bereits sehr weit ausgearbeitete Tarifstruktur SwissDRG detaillierter als die in der Schweiz verwendete Version 6 von APDRG. Sie entspricht nur einem kleinen Teil der Reform der Spitalfinanzierung, die nächstes Jahr stattfinden wird. Die berechtigten und verständlichen Befürchtungen unserer Kolleginnen und Kollegen betreffen weit mehr die anderen Parameter der neuen Spitalfinanzierung als die Tarifstruktur an sich. Die Blockierung eines der am weitesten fortgeschrittenen Elemente der Reform wird die anderen Probleme nicht lösen. Sie wird aber alle bestrafen, die sich auf den Termin vorbereitet haben!

Die Begründungen der Petitionäre dürfen nicht unwidersprochen bleiben:

- Das System SwissDRG wird nur für die akutsomatischen Spitalaufenthalte gelten, nicht jedoch für den Langzeitbereich. In diesem Zusammenhang werden Patienten mit mehreren Krankheiten gut berücksichtigt, da spezifische, angemessen vergütete Gruppen mit komplexen Erkrankungen bestehen.

- In keinem Kanton, in dem diese Art von Verrechnung eingeführt wurde, löste die Entschädigung der Spitäler über ein DRG-System eine besondere Kostensteigerung aus, die sich auf dieses System zurückführen lässt. Hingegen kann die Neuaufteilung der Lasten zwischen Versicherern und Kantonen zu Veränderungen auf der Ebene der Prämien und der Steuerbelastung führen.

- Die allmähliche Verlagerung der Tätigkeit in den ambulanten Bereich - Spital oder Arztpraxen ist eine unausweichliche Realität, wirtschaftlich rationell und wird von den Patienten gewünscht. Damit erhöht sich der Bedarf nach Kommunikation unter dem Gesundheitspersonal, was positiv ist. Noch in diesem Jahr wird eine gemeinsame Studie von $\mathrm{FMH}$ und $\mathrm{H}+$ anlaufen, um diese Bewegungen zu erfassen. Derzeit ist keine Ausdehnung der DRG auf den ambulanten Sektor geplant.
- Das Problem der Finanzierung der ärztlichen Weiterbildung ist erkannt. Das BAG hat eine Plattform aufgebaut und der Bundesrat empfiehlt diesbezüglich eine Motion der Gesundheitskommission des Nationalrats zur Annahme. Übrigens haben zahlreiche «DRG-Kantone» im Budget ihres Erziehungsdepartements bereits die erforderlichen Finanzmittel bereitgestellt.

- Der wirtschaftliche Druck ist mit oder ohne DRG ein unabwendbarer Faktor im Spitalalltag und beeinflusst unsere Arbeit. Wir müssen auch den Nachwuchs für diese Frage sensibilisieren.

- Im Hinblick auf eine angemessene Arbeitsbelastung des Gesundheitspersonals sind gewisse Reorganisationen notwendig, um die Mehrarbeit in die unterbeschäftigten Sektoren zu verschieben.

- Das DRG-System führt oft zu einer Spezialisierung und zur Bildung von Kompetenzzentren. Statt einer Vervielfachung von unnötigen Leistungen muss eher die Gefahr einer Verknappung in der Behandlung der schweren Krankheiten analysiert werden.

- Die Begleitforschung soll auf mehreren Ebenen die Auswirkungen der neuen Finanzierung messen. Dies ist jedoch erst dann möglich, wenn die Umstellung stattgefunden hat! Mehrere Projekte laufen schon dieses Jahr an, damit Ausgangsdaten zur Verfügung stehen.

- Bezüglich der Weitergabe der Daten sind die Tarifpartner zu keiner Einigung gelangt. In dieser Frage muss somit die Bundesbehörde entscheiden, was nächstens der Fall sein wird.

Das Bundesparlament hat das Datum für die Einführung des neuen Systems gemäss den Übergangsbestimmungen des KVG auf den 1.1.2012 festgesetzt. Es liegt nicht in der Kompetenz des Bundesrats, diesen Termin zu verschieben - somit macht es auch keinen Sinn, an ihn zu gelangen. Um ihre eigenen Interessen und jene der Patienten optimal zu vertreten, muss die Ärzteschaft weiterhin eine kritische, aber partizipative und konstruktive Haltung einnehmen - im Einvernehmen mit den Regeln unseres Staates. Ein grundsätzlicher, schlecht begründeter Widerstand, der die objektive Realität ausser acht lässt, schadet unserer Glaubwürdigkeit. 